\title{
REDISEÑAR LAS RELACIONES PEDAGÓGICAS ENTRE TECNOLOGÍA Y ESCUELA. NO SON LAS MÁQUINAS, ES LO QUE PONEMOS EN ELLAS
}

REDESIGN THE PEDAGOGICAL RELATIONSHIPS BETWEEN TECHNOLOGY AND SCHOOL. IT'S NOT THE MACHINES, IT'S WHAT WE PUT IN THEM

DOI: $10.25100 /$ nc.v0i27.10609

Rocío Gómez

Universidad del Valle, Cali, Colombia rocio.gomez@correounivalle.edu.co ORCID: 0000-0003-3077-3533

Julián González Mina²

Universidad del Valle, Cali, Colombia julian.gonzalez@correounivalle.edu.co ORCID: 0000-0002-0766-5772

Recibido: 2 de mayo de 2020

Aprobado: 22 de junio de 2020 
Resumen: el artículo presenta una síntesis de los Semilleros de Tecnologías de Información y Comunicación, una iniciativa para el uso, incorporación y apropiación de tecnologías digitales llevada a cabo en 40 instituciones escolares de Cali (Colombia). Esta iniciativa experimentó un camino diferente. No pensó las tecnologías como máquinas de distribución de contenidos ni las puso en el centro del proceso de aprendizaje. El proyecto asume como principio pedagógico transformador que la clave son los maestros y los estudiantes y su capacidad para crear trabajo y coordinar acciones basadas en el uso de conocimiento y recursos disponibles, localizados y sensibles al contexto.

Palabras clave: Tecnologías de la Información y de la Comunicación, Aprendizaje Asistido por Ordenador, Diseño de Sistemas, Trabajo en Equipo, Trabajo Escolar.

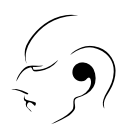

Abstract: the article presents a synthesis of ICT Seedbeds, an initiative for the use, incorporation and appropriation of digital technologies executed in 40 school institutions in Cali (Colombia), which experienced a different route to the options focused on the content and the machine, which grant the technological mediation almost the entire weight of the learning system. The project assumes as a transformative pedagogical principle that the focus is on teachers and students, and on their ability to generate works and coordinate actions based on the recruitment and use of knowledge and resources available, located, sensitive to the context.

Keywords: Information and Communication Technologies, Computer-Assisted Learning, System Design, Teamwork, School Work.

Este trabajo está bajo la licencia Creative Commons BY NC SA 4.0.

¿Cómo citar este artículo? / How to quote this article?

Gómez, R., y González, J. (2020). Rediseñar las relaciones pedagógicas entre tecnología y escuela. No son las máquinas, es lo que ponemos en ellas. Nexus, (27), pp-pp. doi: 10.25100/nc.v0i27.10609 


\section{Introducción}

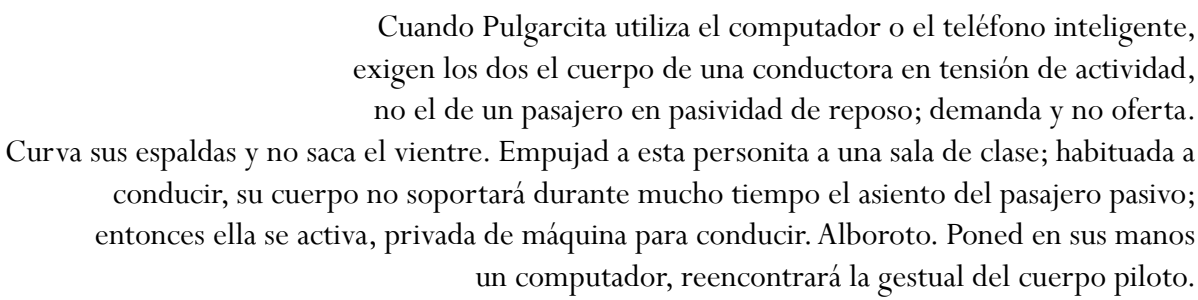

un computador, reencontrará la gestual del cuerpo piloto.

(Serres, 2012)

Las inversiones públicas y privadas orientadas a dotar de tecnología la escuela para mejorar y cualificar los procesos educativos no cesan. Construir infraestructura, favorecer el acceso, formar en el nuevo repertorio técnico a los docentes, y crear contenidos y repositorios ajustados al nuevo sistema técnico son probablemente los cuatro frentes de trabajo usuales cada vez que se pone en marcha un nuevo advenimiento técnico en la educación escolar de masas, en las sociedades contemporáneas. Para cada innovación tecnológica incorporada a la escuela ha habido una panoplia de esfuerzos orientados a la gestión de infraestructura, acceso y dotación de dispositivos, formación de formadores en el nuevo credo técnico y creación de contenidos adaptados al nuevo medio, todo debidamente adobado de prédicas redentoras y promesas. Eso ha ocurrido con el libro de texto, la imprenta escolar y el esténcil, la radio, el cine, la televisión y el video en las aulas. Y en la actualidad, lo hacen las inversiones orientadas intensamente hacia los entornos digitales interconectados en redes.

Llevamos 200 años, desde la expansión de la energía eléctrica, intentando conectar el sistema nervioso central de las personas a máquinas de comunicar contenidos. Y como en anteriores ocasiones, la última avanzada, la de los computadores en la escuela, ha tenido resultados más bien agridulces que no se compadecen con sus costos y sus grandilocuentes promesas (Buckingham, 2008). Impactos limitados de los nuevos dispositivos técnicos en la formación matemática y en el dominio de la lengua propia o de una lengua extranjera. Mejores resultados se obtienen cuando se evalúa, en jóvenes y niños escolarizados, el dominio y uso de las tecnologías informáticas en sí mismas. Resultados poco concluyentes en relación con la formación en ciencias naturales y ciencias sociales. Estos resultados deslucidos se presentan incluso cuando la incorporación del computador y la tableta se hace poniendo en marcha un complejo, robusto, bien pensado y aceitado sistema como el Plan Ceibal en Uruguay. "Los resultados sugieren que el Plan Ceibal no habría tenido un impacto en matemática y lectura. Estos resultados se encuentran en línea con la mayor parte de la literatura sobre el impacto del uso de computadoras en el aprendizaje, la cual encuentra resultados nulos o negativos" (de Melo, Machado, Miranda y Viera, 2013, p.12). 
¿Entonces hay que renunciar a las aventuras tecnoeducativas y sus promesas? ¿Habrá que prepararse para el futuro fiasco de la Inteligencia Artificial en la educación escolar, ahora que la inclusión de computadores parece habernos defraudado? ¿Qué estamos haciendo mal? ¿Cuál es el error, si hay error alguno? ¿Por qué cada avanzada tecnoeducativa nos devuelve una bofetada más o menos sonora que nos despierta de nuestras ilusiones redentoras?

Quizás el error reside en asumir, más o menos automáticamente, los atributos de la máquina como estructuradores del aprendizaje, ignorando el papel crucial de las personas en la creación de saber, en la generación de obras significativas y en la configuración que ocupan las propias máquinas en los procesos educativos. Si se examina en detalle la literatura técnica sobre el impacto de las TIC en educación suele encontrarse, de entrada, una cierta caracterización de las nuevas tecnologías que enfatiza en sus atributos y se los relaciona con algunos cambios deseables en los modos de trabajar, aprender y conocer en la escuela. Términos como automatización, interactividad, conectividad, acceso y flujos de información, gestión de contenidos digitales, velocidad de procesamiento, aumento del poder de cómputo, procesamiento de información y analítica de datos parecen exigir, consistentemente, formas de trabajo y aprendizaje flexibles, disposición a la adaptación rápida a un entorno laboral cambiante, competencias para aprovechar recursos de información y datos crecientes, habilidad analítica para interpretar datos y relacionarlos. De pronto, las pautas y atributos de la máquina se convierten en modelo e ideal educativo. De este modo se incurre en un error que es necesario evitar: asumir que todos los valores y atributos de las TIC son deseables, y que el aprendizaje y trabajo humanos deberían adecuarse rápidamente a ellos ${ }^{3}$. Se olvida que ciertos aspectos de la máquina no sean compatibles con la naturaleza del trabajo escolar y el aprendizaje.

Por ejemplo, el hecho de que las máquinas digitales e informáticas sean pensadas como dispositivos que facilitan ciertas labores intelectuales y físicas - automatizando algunos aspectos engorrosos y difíciles del trabajo humano-, aunado a un sistema escolar que privilegia la transmisión de contenidos, ha producido una consecuencia inesperada: al facilitar la búsqueda de información, al mejorar los procesos de descarga de archivos y al aumentar las posibilidades de manipular y editar fragmentos de datos y documentos, se multiplicaron las prácticas de copiado y pegado de textos. El automatismo técnico de la máquina, unido al énfasis en la transmisión de contenidos del sistema escolar, ha derivado en un crecimiento exponencial del copy y paste en todos los niveles del sistema escolar, provocando una epidemia de plagios que ha sido resaltada en medios de comunicación a lo largo y ancho del mundo.

Sostenemos, entonces, que las nuevas máquinas incorporadas al sistema escolar deben -más que facilitar y ahorrar- aumentar e intensificar el trabajo. Es decir, a diferencia de lo que ocurre con la producción industrial de bienes materiales, en la que la eficiencia de las máquinas se expresa como reducción de los esfuerzos de 


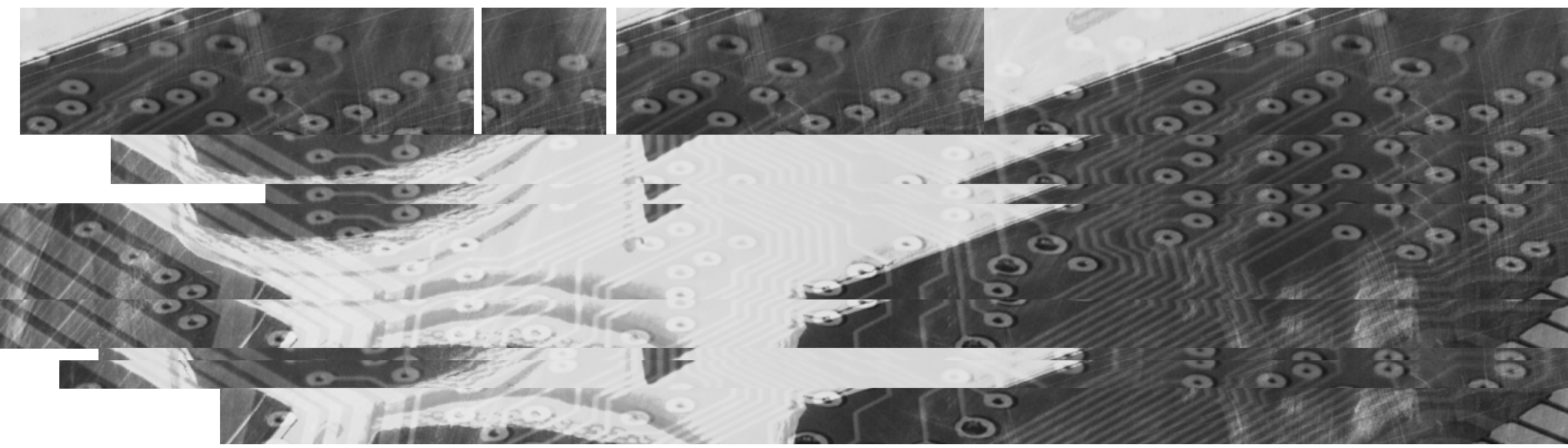

trabajo humano, en el sistema escolar una dinámica similar no es deseable. Como ha señalado Bourdieu (1979), el capital cultural se incorpora, se vuelve cuerpo y hábito, mediante el tiempo de trabajo. El trabajo de crear, entender, producir, leer va transformándose en saber y hábito, y en ese sentido, requerimos que las máquinas y el sistema escolar intensifiquen ese trabajo que produce tanto al sujeto creador como a sus obras o creaciones. Si las máquinas automatizan el trabajo escolar afectan dramáticamente lo esencial de la experiencia educativa. En ese sentido, si hubiera que evaluar el impacto de las TIC en la educación escolar, habría que empezar por preguntarse en qué medida su incorporación aumenta o intensifica el trabajo de creación y producción de obras propias que los agentes educativos ponen en marcha usándolas. Por esa razón, habría que plantearse cómo hacer para que la incorporación escolar de nuevas tecnologías aliente el trabajo humano y modere en ellas su tendencia a automatizar y facilitar tareas ${ }^{4}$. El éxito de la apropiación tecnológica en la escuela se debe medir en términos de su capacidad para favorecer mayor inversión de trabajo creativo en los agentes humanos del sistema escolar.

Este artículo presenta de manera sintética las ideas detrás de una iniciativa de uso, incorporación y apropiación de tecnologías digitales en instituciones escolares que experimentó una ruta distinta a las usuales. En ella, se subraya el uso de las máquinas para complejizar el trabajo humano. Se subordinan algunos aspectos automáticos de estos aparatos a los propósitos de uso no automáticos de los educadores y educandos. Para hacerlo, el proyecto se inclinó por una solución sugerida por Buckingham (2008) hace diez años. Podemos aprender cómo conectar escuela y nuevas tecnologías examinando cómo las personas comunes y, en particular, los niños y jóvenes usan e incorporan las tecnologías en sus vidas no escolares. Al examinar esos usos no escolares de las tecnologías, se puede notar que suelen invertir mucho tiempo y trabajo intensivo en las máquinas, escribiendo, reciclando, rediseñando, comentando y creando piezas que, probablemente, no tienen mucho valor escolar, pero que les resultan esenciales para vivir. En cambio, cuando incorporan esas máquinas en sus clases, se pliegan a los automatismos y producen abundantes plagios y mezclas de dudoso valor escolar y limitado valor para sus propias vidas. 


\section{Cuatro rutas}

Desde enero de 2018, 20 Instituciones Educativas Oficiales (IEO) del municipio de Santiago de Cali (Colombia), participaron en la iniciativa Semilleros TIC, uno de los componentes del programa Mi Comunidad es Escuela ${ }^{5}$. En 2019, 20 IEO adicionales se sumaron a esta iniciativa. Su objetivo fue estimular en docentes y estudiantes la creación intensiva de obras de diverso tipo, apelando al uso de recursos TIC y no TIC disponibles en las escuelas. El proyecto derivó en la creación de un Banco de Aplicaciones 0.0, un total de 116 aplicaciones que atienden problemas de convivencia, democracia, identidad y territorio, así como temas específicos de ciencias sociales, matemáticas, español, entre otros ${ }^{6}$. A lo largo de dos años, el proyecto acompañó a 3.810 personas, entre estudiantes y docentes.

Esta experiencia rechaza tres configuraciones o modos de incorporación de Tecnologías de Información y Comunicación en la escuela, y se inclina por una cuarta. A continuación, se designa cada una de las cuatro configuraciones usando los nombres de marcas corporativas. Cada una de esas marcas permite reconocer, condensar e ilustrar cuatro modos distintos de concebir el lugar de las tecnologías en la producción, circulación y uso de conocimiento y saber escolar.

\section{Configuración 1. Máquinas de distribuir y diseminar contenidos}

El libro, la primera y más eficiente máquina industrial de comunicar contenidos, pautó y modeló las siguientes. Los medios de comunicación industrial más conocidos como la radio, el cine, el teatro educativo, la televisión, los carteles - carteleras-, el video, las infografías y procedimientos visuales de representación gráfica-esquemas, cuadros, mapas mentales-, son hijos más finos y desarrollados de esta configuración: tecnologías dinámicas de representación y diseminación de contenidos. El patrón contenidista se reactualiza en los entornos audiovisuales y digitales, sin dejar de prolongar y expandir la matriz del libro como máquina de diseminación: videojuegos y juegos educativos, Objetos Virtuales de Aprendizaje, enciclopedias interactivas y digitales, televisión educativa tipo Discovery o BBC, o simuladores, quizás la forma menos contenidista de esta configuración tecnoeducativa, no hacen más que extender el proyecto divulgador del libro. Son como libros educativos dinámicos.

En esta configuración la persona es, para decirlo de un modo simple, un pasapáginas, un activador o navegador de los contenidos que circulan a través de la máquina. Vamos a llamar a esta configuración de acuerdo con una de sus formas industriales más populares y conocidas: Encarta-Discovery.

Esta configuración entiende el repertorio técnico como un atractivo repositorio y vehículo de diseminación de contenidos escolares. Y en ella, la figura perfecta del aprendiz es, sin duda, el lector competente y crítico que, eventualmente, podrá convertirse en productor (escritor, creador de obras). 
Configuración 2. Aprender a operar comandos y activar procesos informáticos

En la segunda ruta o configuración, no se trata de contenidos de primer nivel (archivos, documentos que se transmiten al sistema nervioso central de la persona mediante vehículos de contenidos), sino de segundo nivel. Lo que las personas aprenden son procedimientos para operar, crear, diseñar, y archivar contenidos de primer nivel. La forma perfecta de esta configuración son los tutoriales y los cursos para dominar paquetes de programas informáticos.

Es la configuración que implica el dominio operativo del software y en ella la figura que educa es, esencialmente, un instructor -cuando se trata de un agente humano- o un tutorial. El tutorial puede adoptar la forma de libro, de instructor digitalizado en YouTube, de FQS - preguntas frecuentes- o de foros colaborativos en línea. En esta configuración el propósito central consiste en aprender a operar el software hasta terminar en prácticas más o menos profesionales y autónomas de uso. El aprendizaje de código o de lenguajes de programación sería la expresión más elaborada y sofisticada de esta configuración tecnoeducativa. Es decir, la figura perfecta de aprendiz en esta configuración es aquel que pasa de eficiente operador de software a programador.

Le llamaremos Microsoft Office a esta configuración que asume los entornos tecnológicos como oportunidades de entrenamiento y operación de software y paquetes informáticos mediante el dominio de instrucciones.

\section{Configuración 3. Inteligencia artificial y sistemas autónomos}

La tercera, la forma Google / Brainy ${ }^{7}$, aspira a extender las virtudes de la automatización a los procesos de aprendizaje. Intenta desarrollar plataformas cada vez más autónomas, extender tipos de software que se robustecen con el uso, generar entornos basados en inteligencia artificial capaces de adaptarse a las demandas de formación del usuario, entornos digitales que aprenden según acumulan usuarios y se refinan con los aportes de éstos. Plataformas que se anticipan y ajustan a las demandas de uso, corrigen errores y prevén - de manera creciente- toda clase de situaciones de aprendizaje. En esta configuración el profesor se convierte en un acompañante o asistente que ayuda a corregir y afinar la marcha y desarrollo de la interacción plataforma-usuario. La figura perfecta del aprendiz virtuoso en este caso es el hábil gamer, el eficiente ejecutor de videojuegos o el acumulador de mejores y más amplios puntajes y registros, un poco como el famoso y memorioso Ken Jennings, campeón del programa concurso Jeopardy.

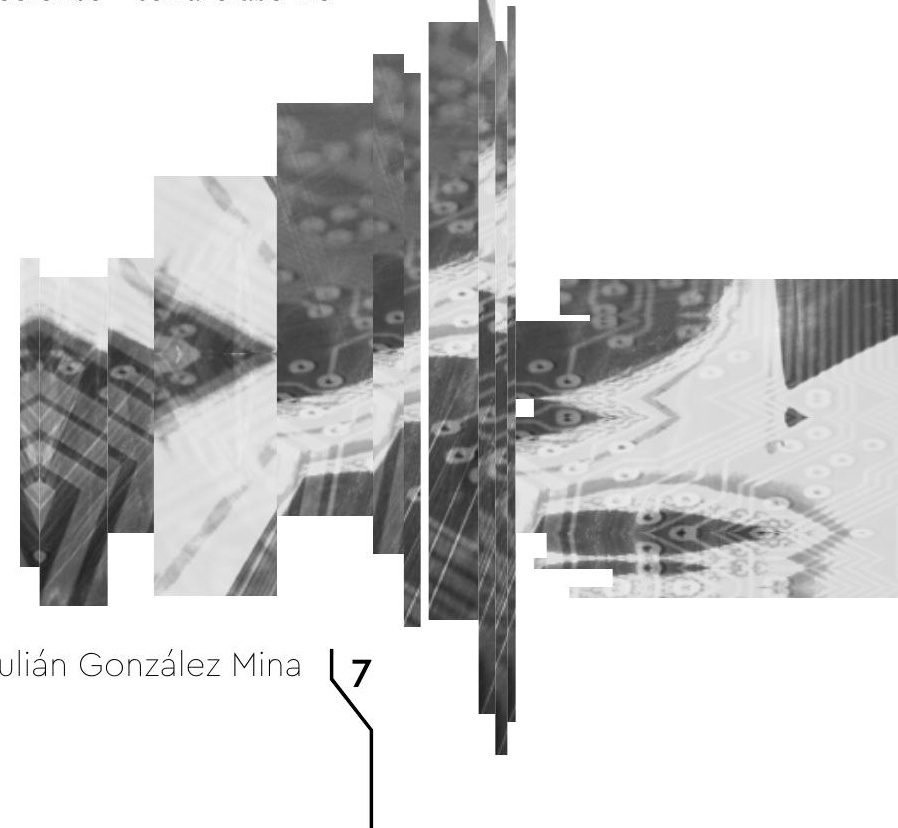




\section{Configuración 4. Programar socialmente y de manera situada las máquinas}

Y finalmente, la cuarta configuración es la forma Uber. Mientras las anteriores configuraciones son fuertemente tecnocéntricas y contenidistas, esto es, conceden a la mediación tecnológica casi todo el peso del sistema de aprendizajes, la forma Uber asume que el centro del sistema está en los usuarios, en el conductor/pasajero debidamente coordinados por la plataforma. En esta configuración lo clave es la generación de recursos, obras y la coordinación de acciones a partir del reclutamiento y uso de aquello que está disponible localmente, incluyendo el repertorio técnico a mano. Es una configuración oportunista: se usa y articula aquello con lo que se cuenta en la situación dada. No hay derivas prefijadas, y los resultados, aunque no son aleatorios, dependen de contingencias y circunstancias específicas.

En esta configuración el aprendizaje óptimo ocurre cuando el repertorio tecnológico y los recursos disponibles se usan para crear obras propias que sólo pueden realizarse tendiendo vínculos con otras personas y procurándose saber mutuamente. Es, además, la única configuración en la que los contenidos, en sí mismos, no son el centro y, en consecuencia, los procesos educativos no quedan apresados en alguna instancia que los suministra -ya sea el docente, la corporación que procura la plataforma o el software, el programador o el dispositivo tecnológico en sí mismo--

En síntesis, las instituciones escolares pueden adoptar la lógica Encarta-Discovery y confiar en que el mejoramiento del acceso a contenidos de calidad es suficiente; pueden cifrar en el modo Microsoft o en el entrenamiento y dominio operativo del software la apuesta formativa en tecnologías; pueden aspirar a costosos y complejos sistemas autónomos y relativamente adaptativos, que prometen personalizar el aprendizaje, ajustar el suministro de contenidos, evaluar los avances de cada estudiante y prever tipos de problemas de saber según las características del usuario. En estos tres casos, se profundiza la dependencia de la institución escolar respecto a las corporaciones que ofrecen los contenidos, el software y las actualizaciones a los servicios informáticos ${ }^{8}$.

La lógica Uber, en cambio, invierte los términos: pone el énfasis en las disposiciones, recursos y posibilidades situados en el entorno, y coordina su utilización. Opera según propósitos específicos definidos por los usuarios contextualmente. En esta lógica, para decirlo de manera esquemática, la inteligencia grande no está en las máquinas sino en los usuarios. 


\section{Inventar los usos de las máquinas: la experiencia de los Semilleros TIC en Mi Comunidad es Escuela}

La modalidad de Semilleros TIC (2018-2019) que se desarrolló en esta experiencia implicó asumir que profesores y estudiantes diseñan procedimientos de uso del repertorio técnico disponible en las IEO de conformidad con proyectos de creación y saber propios. Se trata de adaptar el software de la máquina (esa inteligencia chiquita y acotada que permite automatizar ciertos procedimientos ${ }^{9}$ ) a la inteligencia grande y compleja de las personas.

Es importante señalar que "máquina”, cuando se refiere a Tecnologías de Información y Comunicación, es realmente un enjambre de componentes, modulares, funcionales y relativamente autónomos unos respecto a los otros. Teclados, sensores, visores, puertos, pantallas, comandos para modificar lo que se ve en las pantallas, grabadoras de audio, memorias, linternas, cronómetros, mapas, medidores de distancia, de luz o sonido, etc., son “máquinas" disponibles en la máquina denominada teléfono móvil, computador, tableta o impresora. Si a lo anterior se añade la posibilidad de descargar aplicaciones, software adicional o actualizaciones, la "máquina de máquinas" que es cualquiera de estos dispositivos digitales se multiplica aún más. Cada una de esas máquinas modulares puede desempeñar tareas específicas y diferenciadas en una iniciativa de uso escolar. Por ejemplo, la función Swype del teclado de un teléfono móvil puede usarse para, mediante la rutina de trazar ciertas formas, nombres o figuras en él, generar palabras que se usarán para crear relatos o cuentos (Figura 1).

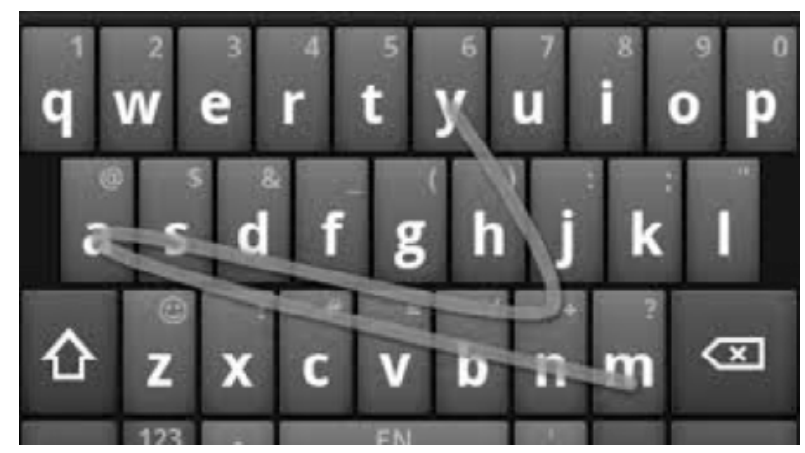

Figura 1. La función swype del teclado sensible de un teléfono móvil puede generar palabras a través de lenguaje predictivo. Esas palabras se usan para abrir la construcción de cuentos de 280 caracteres en una aplicación 0.0 denominada Twittea o Trina Tu Cuento. En esta se especifica y se define el paso a paso: Primero trace las iniciales de su nombre y apellido en el teclado. Seleccione las dos palabras generadas por el teléfono móvil. Esas dos palabras deberán hacer parte del microcuento que escribirá, y no podrá exceder 280 caracteres. Los participantes de Twittea o Trina Tu Cuento tienen 40 minutos para escribir su relato. Luego votarán por los mejores.

Fuente: elaboración propia 
En ese sentido, lo que se ha hecho con Twittea o Trina tu Cuento es programar socialmente el software para que desempeñe funciones que no están inscritas en él, sino en la situación educativa esperada (Figura 1).

En una IEO rural se instaló un teléfono móvil, en una zona donde todo tipo de aves silvestres solían ir a comer. Había granos de cereales, frutas y alimentos varios que atraían a las aves a esta videotrampa que grababa, mediante autofoco, todo lo que se moviera en su campo de registro. Luego, había una cierta expectativa por ver juntos qué había quedado registrado en la videotrampa. Este registro audiovisual era el punto de partida para avanzar en un conjunto de actividades escolares diversas: identificar el tipo de aves, averiguar cuál era su dieta, conocer si se trataba de aves endémicas o migratorias, a qué familias pertenecían, qué diferencias había en sus trinos. Este tipo de videos son un recurso propio y local que se debe leer, interpretar y analizar. Videomove es el nombre con que se designó a un conjunto de procedimientos de uso del video obtenido a través del teléfono móvil para cazar visualmente la vida animal silvestre del entorno y usarle en las clases (Figura 2).

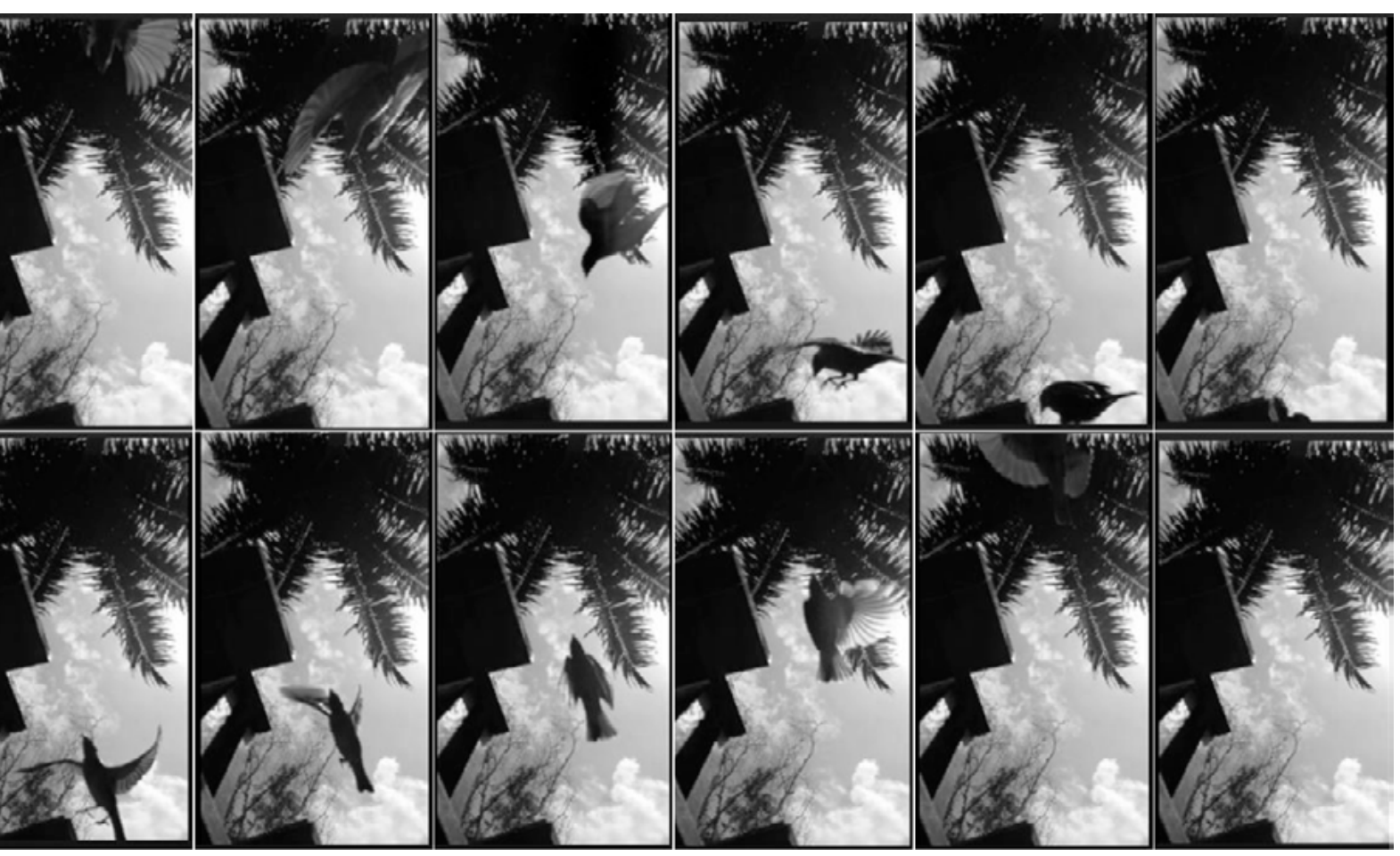

Figura 2. Diez fotogramas de VideoMove. Instantes en que un ave cruza el videograbador de un teléfono móvil que “caza” la presencia de aves silvestres en un comedero. La secuencia se desarrolló entre el minuto 07:57:20 y el minuto 07:58:17. Además del teléfono móvil, se usó un computador, la función Recortes de Microsoft y el visor cuadro a cuatro. Es decir, se usa el repertorio tecnológico no para aprender el software, sino para crear una obra que tiene relevancia escolar. ¿Cuántos niños han examinado con cuidado el aleteo de las aves que cruzan los cielos de sus propios colegios?

Fuente: elaboración propia 
Dicho de otro modo, en los Semilleros TIC del proyecto Mi Comunidad es Escuela, profesores y estudiantes crearon programas de uso (procedimientos de uso) de los programas informáticos y máquinas disponibles. Programaron socialmente estas máquinas. A esos procedimientos de uso se les llamó Aplicaciones 0.0. Semilleros TIC puso en el centro el trabajo que pueden invertir estudiantes y profesores en la producción de obras de relevancia escolar, en la generación de vínculos que procuran formas de convivencia significativas en IEO - harto permeadas por conflictos sociales y personales asociados a las difíciles condiciones de vida de las comunidades y barrios pauperizados de donde provienen- y en los procedimientos de cooperación y colaboración exigidos por el tipo de desafíos y problemas de creación y conocimiento inventados e imaginados por estudiantes y profesores.

Las TIC no operaron como modos de acceder a contenidos, monitorear desempeños o administrar rutinas escolares. Así, estudiantes y profesores fueron conminados a inventar y probar procedimientos y pautas de uso de las tecnologías disponibles en sus escuelas para diseñar situaciones educativas que favorecieran la producción colaborativa de conocimiento, obras y vínculos en la comunidad escolar. Mejores y más potentes computadores no son suficientes para hacer transformaciones educativas emancipadoras sin concebir formas organizativas y situaciones que hagan realmente creativo y vigoroso el trabajo educativo. No hay tecnología sin situación de uso. En Semilleros TIC consideramos que en los procedimientos pedagógicos para enriquecer esas situaciones está la clave educativa.

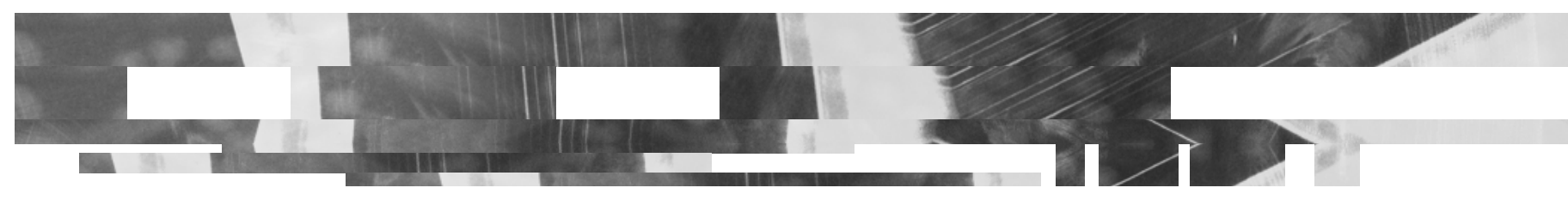

Esta perspectiva se sustenta en la tradición del Grupo de Educación Popular de la Universidad de Valle, según la cual el centro de toda apuesta pedagógica está en al menos dos posturas. La primera, partir de un marcado desafío a la razón dualista y a las hipótesis de separabilidad total (Castoriadis, 1989; 1991; 1997) que suelen diferenciar los elementos constitutivos de lo real y al mismo tiempo señalar la preeminencia o jerarquía de algunos de los elementos diferenciados, como cuando se distingue analíticamente entre saber científico/saber popular, práctica/teoría, texto/contexto, mente/cuerpo, entre otros. En la segunda postura es necesario desconfiar de las convenciones que prescriben quién sabe y quién no sabe. Por ello en Semilleros TIC el punto de partida educativo estuvo en explorar imaginativamente los usos que los sujetos hacen de la tecnología y en la manera cómo esos usos pueden tener réditos educativos en el contexto escolar y no escolar. Nos apoyamos en los resultados de investigaciones previas que hemos realizado sobre el tema, algunas de ellas empíricamente fundadas (Gómez y González, 2005; Gómez y González, 2008; 
Gómez Zúñiga, 2012; Gómez et al., 2016; González Mina, 2018; Gómez Zúñiga, González Mina y Rueda Ortiz, 2019) sobre los usos sociales de los nuevos entornos tecnológicos y las formas en que jóvenes urbanos integrados construyen maneras particulares de hacer política y participar empleando las nuevas tecnologías; sobre los modos de poetización de la vida que sujetos diversos construyen a través de Facebook; sobre las distintas maneras de cognición en niños videojugadores, o sobre los procesos de programación social de las tecnologías que se pueden generar con un grupo de jóvenes en el contexto de una biblioteca pública, entre otras.

Desde esta perspectiva planteamos la relación entre nuevas tecnologías y escuela como un escenario de diálogo de saberes en el que estudiantes y profesores son asumidos como sujetos de conocimiento, no simples usuarios que se entrenan en el uso "correcto" de software y paquetes educativos. Al poner el énfasis en los usos que las personas hacen de las tecnologías y en la capacidad de innovación y creación que allí radica, la ecuación pedagógica se transforma, cesa la centralidad de las máquinas.

\section{Pensar las máquinas y repensar la pedagogía}

Con respecto a la relación entre escuela y tecnologías, la pedagogía suele asumirse como un proceso de construcción de ambientes para el aprendizaje o como el conjunto de principios que animan el diseño de actividades para capacitar a los aprendices en las competencias requeridas por el plan de estudios. La pedagogía también es vista como una disciplina que trabaja sobre las técnicas y materiales para enseñar. Otro enfoque asume la pedagogía como un conjunto de procesos que optimizan la comunicación, en el sentido más instrumental y pobre del término, aquel que popularizaran Shannon y Weaver (Shannon, 1948; Shannon y Weaver, 1949): hay un "emisor" (profesor/a) que produce "mensajes"(enseñanzas), con unos "medios" (instrumentos y materiales) para unos "receptores" (alumnos/as) y a través de un conjunto de canales. Mejía (2012) señala al menos tres taxonomías que clasifican la pedagogía bajo diferentes enfoques. La Pedagogía Transmisionista, cuyo objeto es trasladar a los aprendices el conocimiento y entrenarlos en los valores y comportamientos "adecuados". La Pedagogía Activa, cuyo objeto de trabajo es diseñar actividades pedagógicas que promuevan la constitución de los aprendices como sujetos responsables de su propio desarrollo. La Pedagogía de la Tecnología Educativa, cuyo objeto de trabajo es el diseño técnico de procesos pedagógicos que refuercen los aprendizajes, utilizando tecnologías y materiales que articulen los objetivos de la instrucción con resultados medibles a través de indicadores.

Nuestra perspectiva se ancla en Freire $(1970 ; 1978 ; 2014)$, quien transformó el pensamiento educativo al cuestionar las pedagogías que, centradas en la entrega de los conocimientos, terminan por legitimar ideologías opresoras, bajo un manto de neutralidad. Al plantearse desde un punto de vista tecnocrático (transferencia de tecnologías, disminución de la brecha tecnológica, etc.), muchas de las propuestas que establecen la relación entre escuela y tecnologías siguen -aún hoy-vinculándose 
con las comunidades educativas de una manera bancaria y vertical, invisibilizando el sesgo ideológico que perpetua la lógica de quién sabe (las corporaciones tecnológicas, los asesores externos, los técnicos, los ingenieros, los asesores externos) y quién no sabe (los docentes, los estudiantes). El reto es plantear un diálogo que implica presencia activa de agentes humanos y no humanos que se confrontan e interpelan. Potenciar "el cruce de voces, sin que ninguna de ellas tenga el privilegio de silenciar o negar ninguna de ellas" (Ghiso, 2013, p.17). Desde una perspectiva freiriana, el diálogo de saberes sólo puede emerger cuando la cultura de los participantes es suficientemente reconocida y valorada; de ahí que Semilleros TIC se plantea como una estrategia situada, en la que las culturas y contextos de estudiantes y profesores se consideran como punto de partida y escenario desde donde plantear iniciativas para tramitar los conflictos y las diferencias (Martín Barbero, 1987).

En relación con las tecnologías, este punto de vista tensiona el autoritarismo de las agencias productoras de software que se arrogan el derecho de ser las únicas llamadas a programar los usos de las máquinas. En Semilleros TIC consideramos que es posible explorar de manera consistente y creativa usos distintos de las tecnologías para aprovechar lo que realmente educa y transforma la experiencia de las personas: el trabajo humano. No se trata de máquinas y software en sí mismos, sino de las diversas formas de trabajo y uso que en, con y más allá de las máquinas, disponen maestros y estudiantes para educarse mutuamente mediatizados por el mundo (Freire, 1970). El reto es dinamizar una labor de imaginación -en el sentido castoriadiano del término (Castoriadis, 1997)-, esto es la invención poética y creación que no está programada en las máquinas. Semilleros TIC intentó dinamizar esas prácticas de programación social de nuevas tecnologías (Gómez Zúñiga, González Mina y Rueda Ortiz, 2019), una labor que -sin que reciba ese nombre- millones de personas realizan todos los días, como nos permitió comprender Rheingold (2004) cuando hace diez años señaló la importancia de atender el funcionamiento de lo que llamó multitudes inteligentes.

\section{Escuelas y Nuevas Tecnologías de la Información y la Comunicación}

Muchas de las escuelas públicas actuales cuentan con oportunidades inéditas para realizar experimentos y exploraciones creativas a bajo costo, reduciendo riesgos y usando abundantes recursos que, digitalmente, pueden editarse, conectarse y ensamblarse gracias a algunos de los repertorios tecnológicos de los que disponen. "Recursos", por cierto, no refiere únicamente a objetos, dispositivos técnicos o dinero. Refiere tanto a aquello que, producido o no por la comunidad escolar, está disponible digitalmente, como a todo aquello que puede digitalizarse parcial o totalmente. Y lo que puede digitalizarse total o parcialmente son tanto entidades materiales físicas y tangibles que no son producidas por la comunidad escolar, hasta aquellas que son manifestación física y tangible del trabajo que hacen las personas. Pero los recursos más valiosos provienen de la propia experiencia y capacidad de trabajo humano y vivo de quienes hacen parte de las comunidades escolares. La importancia 
La perspectiva educativa crítica de Semilleros TIC asume una mirada no instrumental de las tecnologías y considera a la experiencia como un laboratorio pedagógico para explorar y experimentar con las relaciones entre escuela, entornos tecnológicos y comunidades. A eso hemos llamado “innovar desde abajo” (Gómez Zúñiga, González Mina y Rueda Ortiz, 2019).

Al relativizar la centralidad de las máquinas, no se abandona su uso en las instituciones educativas sino que, más bien, se pone el énfasis en otro aspecto: aprender a usarlas en la escuela comprendiendo cómo son usadas fuera de ella por las nuevas generaciones para comunicar, crear, reciclar y expresarse (Piscitelli, 2009; Serres, 2012), y de qué manera esas formas de uso revelan claves para el trabajo educativo que se pretende liberador y crítico. Comprendiendo cómo las usan, la escuela entiende también quiénes son y de qué manera experimentan la seducción y el goce de conocer y saber a través de ellas. El principio educativo puede resumirse así: explorar e innovar en los usos de las máquinas de comunicar, procurando situaciones en las que los usuarios - miembros de la comunidad educativa- sean intensos productores colaborativos, corporalmente comprometidos y afectivamente implicados. Si los términos parecen demasiado generales, y en cierto sentido poco operativos, vale la pena subrayar que eso es lo que hacen los usuarios todos los días empleando cientos de aplicaciones, plataformas de redes sociales, juegos en línea y sitios web: colaboran, producen, se comprometen corporal y afectivamente, y crean recursos. Sin embargo, esas mismas personas que, por fuera del entorno escolar, son hábiles y juguetones usuarios al usar las nuevas tecnologías, en la escuela parecen vivir de manera fuertemente instrumental y reducida sus relaciones con las máquinas. Superar esa brecha es el desafío.

\section{Lógica 0.0 como principio pedagógico de Semilleros TIC}

Hemos indicado que, en Semilleros TIC, lo significativo no es la máquina sino el trabajo humano que se estimula y potencia. Llamamos lógica 0.0 a ese sentido educativo que sustenta los laboratorios de innovación propuestos en esta iniciativa, según el cual el centro está en la capacidad para conectar contenidos escolares y no escolares, uso de herramientas tecnológicas de todo orden, estrategias pedagógicas, dispositivos y espacios, con la creatividad, los saberes y habilidades de los sujetos (estudiantes y profesores).

Los Semilleros TIC se mueven en un plano pedagógico distinto al de los medios e instituciones desarrolladoras de contenidos escolares. Ponen el énfasis en la innovación, en los usos -lógica 0.0-, y aspiran a que esos usos se puedan articular con contenidos disponibles en diferentes entornos y medios: los OVA (Objetos Virtuales de Aprendizaje), una clase convencional, un libro, un microscopio en un laboratorio de biología, la clase de arte o historia, las ecuaciones en matemática y cálculo, o los ejercicios de química. Su problema no son los contenidos digitales (de hecho, la disponibilidad y acceso a éstos ha crecido de notablemente). La lógica 
0.0 busca crear recursos educativos recuperando y conectando saberes e intereses de los sujetos con "recursos educativos" preexistentes. Adicionalmente, esta lógica asume también como recursos aquellos que se encuentran en el espacio local: el barrio, la vereda, la escuela, las narrativas de los jóvenes, los modos de contar y cantar, las imágenes que profesores y estudiantes sintetizan de camino a la casa, las conversaciones y relatos que hacen para vivir, los modos de moverse y usar sus cuerpos y, sobre todo, en lo que no podrían imaginarse sin arriesgarse a simular.

Las Aplicaciones 0.0 son un conjunto de instrucciones y procedimientos creados por los propios docentes y estudiantes que favorecen el uso de las máquinas -digitales y no digitales- disponibles en las IEO para realizar obras que producen acciones y aprendizajes relevantes y significativos para sus participantes. No son actividades escolares, ni tareas, ni juegos. Son catalizadores o motores - pautas de acción-que sirven para conectar máquinas disponibles en el entorno escolar, intención educativa y acciones significativas que ponen en marcha los participantes. Esas acciones derivan en obras que potencian disposiciones y capacidades de los sujetos y que son educativamente relevantes. Las Aplicaciones 0.0 buscan articular máquinas de viejo y nuevo tipo, recursos disponibles en el entorno escolar y tareas de resolución de problemas cuyo alcance puede preverse, pero no anticiparse completamente.

Las Aplicaciones 0.0 están -literalmente- "por encima" del software, en tanto se diseñan y ejecutan usando los softwares existentes, pero modelando y moldeando su uso; también están "por debajo" del uso del software porque, en muchos casos, proponen una ejecución distinta a la original o logran que ese software haga parte de una iniciativa pedagógica no necesariamente prevista por sus creadores.

Las Aplicaciones 0.0 conservan de las aplicaciones electrónicas el carácter de interfaces que conectan mundo offline y online. Pero, a diferencia de éstas, no son completamente tecnológicas. Consideran una combinación de máquinas de viejo y nuevo tipo, y materiales de uso regular en el sistema escolar como lápices, libros o tableros. Suponen, además, el uso creativo de la diversidad de recursos disponibles en los contextos escolares y abarcan tanto contenidos curriculares como el tratamiento de situaciones que exigen cierta convergencia interdisciplinar de saberes y conocimientos. Apelan al uso creativo de dichos recursos para hacer nuevas comprensiones de problemas y comunicarlas a otros.

Las Aplicaciones 0.0 aportan al desarrollo de aprendizajes escolares diversos disciplinares y no disciplinares-, favorecen el uso de máquinas y dispositivos técnicos disponibles en las IEO e incrementan y diversifican el trabajo que los sujetos invierten en las actividades escolares. Parten de demandas concretas de los participantes, son de fácil uso y comprensión, pretender ser ricas emocionalmente (sorprendentes, inesperadas, entretenidas, bellas), promueven el trabajo en equipo e implican una amplia gama de soluciones. 
La experiencia ganada nos permite distinguir, además, entre Aplicaciones 0.0 de lenta maduración y Aplicaciones 0.0 de rápida maduración. Esto es, aplicaciones que requieren en su proceso de ejecución más tiempo para lograr los objetivos pedagógicos previstos, frente a otras que se desarrollan de una manera mucho más veloz. Cada una tiene su utilidad y pertinencia de acuerdo con las condiciones particulares de las instituciones educativas.

\section{Conclusiones}

Programar los usos del repertorio técnico disponible en las escuelas, atendiendo a las promesas que un régimen extendido y barato de simulaciones digitales permite, es una oportunidad que de ninguna manera debería ignorarse. Con la experiencia de Semilleros TIC logramos que dispositivos técnicos que estaban más bien guardados o subutilizados en las IEO comenzaran a emplearse para producir obras de relevancia escolar y social. Tomar conciencia de que esos usos pueden ser pensados, diseñados, testeados y programados de manera conjunta y colaborativa entre estudiantes y profesores, puede ser una manera valiosa de salirle al paso a las continuas amenazas de obsolescencia, subutilización y dependencia de las escuelas respecto a un régimen permanente de licenciamientos y costosos modelos corporativos de actualización, tanto del software como del hardware. Pero además es un modo de romper con el puro entrenamiento y aprendizaje del software en sí mismo. Usar Excel con el fin de construir las cuentas necesarias para distribuir 30 casas simuladas en un mapa digital del barrio vía Google Street View, indispensable para ilustrar un relato de ciencia ficción de una página, puede ser más útil y eficaz que aprender Excel en sí mismo.

Con Semilleros TIC pusimos en marcha una experiencia educativa transformadora en la que profesores y estudiantes se hicieron cargo de su propio proceso de enseñanzaaprendizaje al encontrar rutas inesperadas para resolver problemas expresivos y estéticos, conceptuales y técnicos, generados por la Aplicación 0.0., tal como lo hacen todos los días miles de millones de personas al usar los dispositivos digitales dentro y fuera de las escuelas. La lógica 0.0 no hace más que formalizar y reflexionar esa práctica social infusa y común de adaptación, apropiación, reutilización y transformación del repertorio técnico disponible a las necesidades y demandas de la vida ordinaria. Esa es la apuesta pedagógica de Semilleros TIC. 


\section{Notas}

${ }^{1}$ Docente del Instituto de Educación y Pedagogía, Universidad del Valle. Comunicadora Social (Universidad del Valle), Doctora en Educación de la Universidad Pedagógica Nacional. Miembro del Grupo de Investigación en Educación Popular.

${ }^{2}$ Docente de la Escuela de Comunicación Social-Periodismo, Universidad del Valle. Comunicador Social y Doctor en Psicología de la Universidad del Valle. Miembro del Grupo de Investigación en Periodismo e Información.

${ }^{3}$ Para una referencia que nos prevenga de este tipo de automatismos recomendamos, a pesar de las objeciones y reparos que merece el catastrofismo de Carr, Superficiales (2013), un estimulante ensayo sobre el impacto de los nuevos entornos tecnológicos en la erosión de los hábitos de lectura profunda y pausada heredada de la cultura del texto y el libro impreso.

${ }^{4}$ Lo ideal es construir una generación de máquinas educativas que, antes que facilitar el trabajo de creación, lo complejicen, intensifiquen y exijan. Esas máquinas ya existen, pero no están inscritas dentro de los sistemas educativos. Las plataformas de redes sociales y las máquinas de videojuego - antes que facilitar- exigen y fuerzan a las personas a compartir, producir e invertir trabajo en ellas para obtener réditos afectivos, sociales y comunicativos.

${ }^{5}$ Mi Comunidad es Escuela (2018-2019) fue un programa de la Alcaldía de Cali (Colombia) orientado a fortalecer la calidad y pertinencia de los procesos educativos de las Instituciones Educativas Oficiales (IEO) rurales y urbanas de Santiago de Cali, priorizando su gestión en los sectores más vulnerables de la ciudad, denominados Territorios de Inclusión y Oportunidades. El programa comprendió cinco componentes: Cali con Educación inicial (primera infancia); Cali con Escuelas Dignas y Seguras (mejoramiento de la infraestructura existente y construcción de nuevas aulas); Cali con Calidad y Pertinencia Educativa; La Escuela Es Mi Comunidad; Cali con Instituciones Fortalecidas con Tecnología. El programa se concentró en 45 IEO, 6.000 directivos docentes, docentes y administrativos, y 172.000 estudiantes con sus familias. En el marco de dos componentes (Cali con Calidad y Pertinencia Educativa, y La Escuela es Mi Comunidad), la Universidad del Valle operó como aliado académico para la ejecución y desarrollo de seis proyectos: Semilleros TIC, Mejoramiento de la Gestión Curricular, Fortalecimiento de Competencias Básicas, Fortalecimiento de Competencias Básicas desde las Artes y la Cultura, Formación en iniciación y sensibilización artística, y Estrategia de Comunicación para el Fortalecimiento de la Gestión Escolar.

${ }^{6}$ Semilleros TIC configuró un Banco de Aplicaciones 0.0, disponible en la siguiente dirección: (https://bit.ly/2NoDKz4). También existe un canal de YouTube en el que se pueden encontrar algunas videoguías de las Aplicaciones 0.0 (https://bit.ly/2BERJOo) y un Repositorio de Recursos Digitales (https://bit.ly/3eyC8yu) que reúne herramientas que pueden ser usadas en el aula de clase.

${ }^{7}$ Un sistema de inteligencia artificial diseñado como asistente educativo, que ajusta los contenidos y tareas que debe aprender y resolver el usuario según su desempeño en las tareas previas. Un poco, a cada quien según su capacidad.

${ }^{8}$ En las IEO donde se desarrolló la experiencia: una porción importante del equipamiento técnico -tabletas, computadores, tableros digitales- estaba guardada o no se usaba porque los paquetes informáticos se habían desactualizado, los profesores no dominaban el software, no sabían cómo asociar las máquinas a las asignaturas y rutinas escolares concretas, o porque los aparatos no eran totalmente funcionales. Sincronizar actualizaciones (actualización del software, hacer mantenimiento al hardware, actualizar a los maestros en los programas informáticos y determinar los modos de uso en relación con asignaturas concretas) parece costoso. Cualquier pérdida de sincronía entre estas actualizaciones se traduce en equipamientos bajo llave, reducción del uso y ruinosas obsolescencias. Nuestra propuesta, en cambio, como se verá más adelante, al centrarse en los recursos disponibles, activos y a mano, y al relativizar la relevancia de la máquina en sí misma, libera las posibilidades de uso 
más allá de las actualizaciones y sincronizaciones.

${ }^{9}$ La automatización de las máquinas de procesamiento se basa en la especificación y acotamiento de la tarea a realizar (Manovich, 2005), de modo tal que, por ejemplo, un dispositivo para el reconocimiento facial o para jugar Go o Ajedrez harán con creciente eficiencia esa "tarea especificada”, pero no podrán hacer otras tareas inespecíficas. La inteligencia social humana, en cambio, opera en condiciones muy borrosas, poco especificadas y débilmente parametrizadas. Enactúa consistentemente y de manera oportunista, como saben recordarnos Varela y otros (Varela, 1990; Varela, Thompson y Rosch, 1992; Varela, 2000; Thompson y Varela, 2001). Por eso hablamos de la inteligencia chiquita de las máquinas y la inteligencia grande de las personas. Arvind Narayanan (2019), profesor de Ciencias de la Computación de la Universidad de Princeton, señala que bajo el término "inteligencia artificial” se están nombrando tecnologías muy diferentes entre sí, con desarrollos desiguales. Indica que los algoritmos destinados a identificar y reconocer perceptualmente patrones para, por ejemplo, hacer diagnósticos médicos, clasificar imágenes o señalar similitudes faciales funcionan bastante bien, "porque no hay ambigüedad en estas tareas" (El Diario, 2019, párrafo 6). El segundo grupo, el que hace juicios de valor - por ejemplo, identificar si un correo es spam o no, si hay razones para cerrar una cuenta de Facebook porque promueve pornografía o contenidos racistas- es menos eficiente. Y sobre los algoritmos que prometen identificar y predecir conductas y comportamientos a partir de ciertos indicios -identificar si alguien miente a partir de los patrones de parpadeo de los ojos, o posibilidades de que alguien pueda cometer un acto terrorista-, sencillamente declara que son una auténtica estafa.

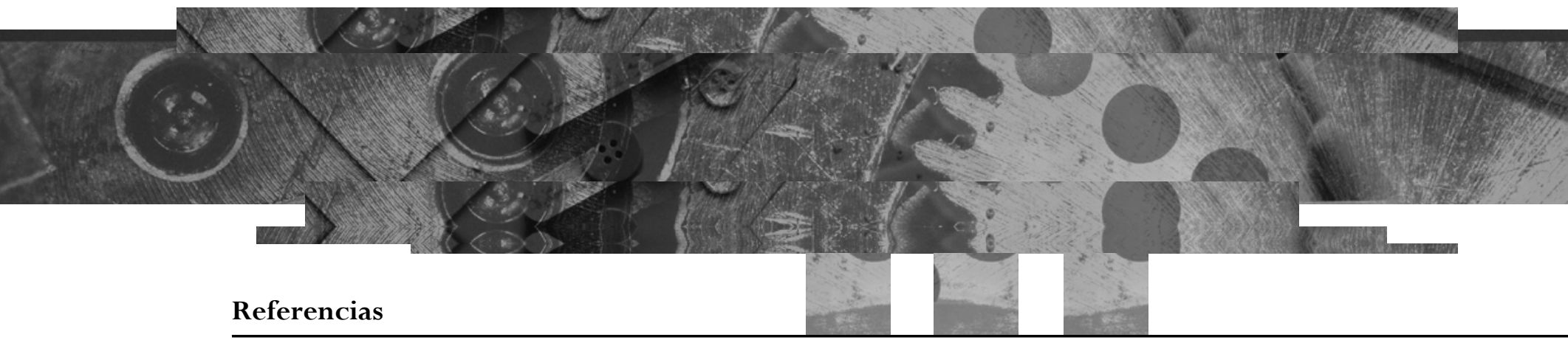

Bourdieu, P. (30 de noviembre de 1979). Los Tres Estados del Capital Cultural. Sociológica, (5), 11 17.

Buckingham, D. (2008). Mas allá de la tecnología: aprendizaje infantil en la era de la cultura digital. Buenos Aires: Manantial.

Carr, N. (2013). Superficiales ¿Qué está haciendo internet con nuestras mentes? México D.F.: Taurus.

Castoriadis, C. (1989). La institución imaginaria de la sociedad (vol. 1 y 2). Barcelona: Tusquet.

Castoriadis, C. (1991). Reflexiones sobre el desarrollo y la racionalidad. En F. Viviescas, y F. Giraldo (Eds.), Colombia: El despertar de la modernidad (pp. 90-111). Bogotá: Foro Nacional Por Colombia.

Castoriadis, C. (1997). Ontología de la Creación. Bogotá: Ensayo y Error.

del Castillo, C. (17 de diciembre de 2019). Los científicos se cansan de la moda de la Inteligencia Artificial y piden prohibir algunos de sus usos: "No se sostienen". El Diario. Recuperado de https: / / www. eldiario.es / tecnologia / cientificos-Inteligencia-Artificial-prohibirsostienen_0_975002803.html

de Melo, G., Machado, A., Miranda, A., y Viera, M. (2013). Impacto del Plan Ceibal en el aprendizaje. Evidencia de la mayor experiencia OLPC. Instituto de Economía, Facultad de Ciencias Económicas y de Administración, Universidad de la Republica, Uruguay. Montevideo: IECON.

Freire, P. (1970). Pedagogía del oprimido (duodécima ed.). Madrid: Siglo XXI de España Editores. 
Freire, P. (1978). La educación como práctica de la libertad. Bogotá: América Latina.

Freire, P. (2014). Pedagogía de la esperanza: un reencuentro con la pedagogía del oprimido. México, D.F.: Siglo XXI.

Ghiso, A. (2013). Formar en investigación desde la perspectiva de la educación popular. En L. Cendales y M. R. Mejía (Eds.), Entretejidos de la educación popular en Colombia (pp. 99-130). Bogotá: Ediciones desde abajo.

Gómez Zúñiga, R. (2012). Procurarse sentido en la ciudad contemporánea. Jóvenes urbanos integrados y nuevos repertorios tecnológicos. Cali: Programa Editorial Universidad del Valle.

Gómez Zúñiga, R., González Mina, J., y Rueda Ortiz, R. (2019). Jóvenes, máquinas y software: reinventar los usos para educar mejor. Cali: Programa Editorial Universidad del Valle.

Gómez, R., y González, J. (2005). Pantallas Reflexivas: Reinventar la Casa y Domesticar las Pantallas Audiovisuales. In VVAA, Los niños y la televisión. Bogotá: Comisión Nacional de Televisión, Programa de investigaciones académicas sobre televisión.

Gómez, R., y González, J. (2008). Tecnologías y malestar urbano entre jóvenes: la celebración de lo inútil y la emergencia del trabajo liberado. Revista Nómadas, (28), 82-93.

Gómez, R., González, J., Rueda, R., Valencia, V., Giraldo, D., Angulo, P., ... Velasco, J. (2016). Facebook como obra mundana. Poetizar la vida y recrear vínculos personales. Cali: Programa Editorial Universidad del Valle.

González Mina, J. A. (2018). Niños que videojuegan, videojuegos que estructuran tiempos. Cognición en los bordes del tiempo irreversible. Recuperado de http://revistas.univalle.edu.co/omp/index. php/programaeditorial/catalog/view/148/7 0/1317-2 ed.). Cali: Programa Editorial Universidad del Valle.

Manovich, L. (2005). El lenguaje de los nuevos medios de comunicación. La imagen en la era digital (primera edición en inglés, 2001 ed.). Barcelona: Ediciones Paidós. (Ó. Fontrodona, Trad.).

Martín-Barbero, J. (1987). De los medios a las mediaciones. Comunicación, cultura y hegemonía. México: Gustavo Gili.

Mejía, M. R. (2012). Educaciones y Pedagogías críticas desde el sur. Bogotá: Cooperativa Editorial del Magisterio.

Narayanan, A. (19 de noviembre de 2019). How to recognize AI snake oil. Recuperado de: https:// www.cs.princeton.edu/ arvindn/talks/MIT-STS-AI-snakeoil.pdf

Piscitelli, A. (2009). Nativos Digitales: dieta cognitiva, inteligencia colectiva y arquitecturas de la participación. Buenos Aires: Santillana.

Rheingold, H. (2004). Multitudes inteligentes: la próxima revolución social. Barcelona: Gedisa.

Serres, M. (2012). Pulgarcita. Barcelona: Editorial Gedisa.

Shannon, C. (1948). A Mathematical Theory of Comunication. The Bell System Techical Journal, 27, $379-423$.

Shannon, C., y Weaver, W. (1949). The mathematical theory of communication. University of Illinois Press.

Thompson, E., y Varela, F. (2001). Radical embodiment: neural dynamics and consciousness. TRENDS in Cognitive Sciences, 5(10), 418-425.

Varela, F. (1990). Conocer. Las ciencias cognitivas: tendencias y perspectivas. Cartografía de las ideas actuales. Barcelona: Gedisa.

Varela, F. (2000). El fenómeno de la vida. Santiago de Chile: Dolmen.

Varela, F., Thompson, E., y Rosch, E. (1992). De cuerpo presente. Las ciencias cognitivas y la experiencia humana. Barcelona: Gedisa. 